\title{
Classifying topoi and the axiom of infinity
}

\author{
ANDREAS Blass \\ In Memory of Evelyn Nelson
}

\begin{abstract}
Let $\mathscr{S}$ be an elementary topos. The axiom of infinity, asserting that $\mathscr{S}$ has a natural numbers object, is shown to be necessary-sufficiency has long been known-for the existence of an object-classifying topos over $\mathscr{S}$.
\end{abstract}

In the known constructions [1, 4, 6] of classifying topoi for geometric theories, it is always assumed that the base topos $\mathscr{P}$ satisfies the axiom of infinity. The purpose of this note is to show that this assumption is necessary. We show that, if a certain very simple geometric theory $T$ has a classifying topos over $\mathscr{I}$, then $\mathscr{S}$ has a natural numbers object. We also show, using well-known methods $[1,4,6]$, that the existence of a classifying topos for $T$ follows from the existence of an object classifier, i.e., a classifying topos for the one-sorted geometric theory having no non-logical symbols and no axioms, the theory whose models in any category are just the objects of that category. Thus, our main result, which answers a question posed in [5], can be stated as follows.

THEOREM. Suppose that there exists an object classifier over the elementary topos $\mathscr{S}$. Then $\mathscr{S}$ has a natural numbers object.

Our notation and terminology will be standard [4].

Let $T$ be the one-sorted geometric theory having one constant symbol $a$, one unary function symbol $s$, no other non-logical symbols, and no axioms. Thus, a $T$-model in any topos (or in any category with a terminal object 1 ) is simply a structure $\mathscr{A}=\left(A, \varrho_{\mathscr{A}}, \mathfrak{1}_{\mathscr{A}}\right)$ where $A$ is an object and $a_{\mathscr{A}}: 1 \rightarrow A$ and ${ }_{{ }^{\prime}}: A \rightarrow A$. (We shall omit the subscripts on a and whenever no confusion can arise). A homomorphism of $T$-models is a morphism between the underlying objects that commutes with the $o$ and $s$ morphisms. A natural numbers object is simply an initial $T$-model. A weakly initial $T$-model (in any topos), i.e., a $T$-model having

Presented by F. E. J. Linton. Received October 30, 1987 and in final form February 24, 1988. 
at least one homomorphism to every $T$-model, will be called a weak natural numbers object. The following lemma is based on ideas from [3, §5] (see also [4, \$6.1]); the same idea, but using external rather than internal completeness, occurs in the proof of the adjoint functor theorem.

LEMMA. If an elementary topos $\mathscr{S}$ has a weak natural numbers object, then it has a natural numbers object.

Proof. Let $\mathscr{A}=(A, 0,6)$ be a weak natural numbers object. Consider the following formula $\phi(z)$ in the Mitchell-Bénabou language [4, §5.4]:

$$
\forall x\left[a \in_{A} x \wedge \forall y\left(y \epsilon_{A} x \rightarrow_{1}(y) \epsilon_{A} x\right) \rightarrow z \epsilon_{A} x\right]
$$

where $x$ is of type $\Omega^{A}$ and $y$ and $z$ are of type $A$. (The formula "says" that $z$ belongs to every sub- $T$-model of $\mathscr{A}$.) The interpretation $|\phi(z)|$ of this formula is a morphism $A \rightarrow \Omega$, the characteristic morphism of a certain subobject $N$ of $A$. Easy calculations show that $N$ is the universe of a sub- $T$-model $\mathcal{N}=\left(N,{ }^{\circ},{ }^{a} \mathcal{N}\right)$ of $\mathscr{A}$ and that the universe of every sub-T-model of $\mathscr{A}$ includes $N$. Since $\mathscr{A}$ is a weak natural numbers object, so is every sub-T-model of $\mathscr{A}$, in particular $\mathcal{N}$. To show that $\mathcal{N}$ is a natural numbers object, consider any two homomorphisms from $\mathcal{N}$ to any $T$-model. Their equalizer is a sub-T-model of $\mathcal{N}$, hence is all of $\mathcal{N}$ by the minimality property of $\mathcal{N}$, so the two homomorphisms are equal, as desired.

The following proposition, though rather easy, is the core of our argument, as it connects classifying topoi with natural numbers objects. In the statement of the proposition, $T$ still denotes the theory defined above, although, as the referee pointed out, the lemma, the proposition, and Remark 2 at the end of the paper all remain correct, when suitably reformulated, for any algebraic (or even essentially algebraic) theory.

PROPOSITION. Suppose $T$ has a classifying topos $\mathscr{E}$ over the topos $\mathscr{S}$. Then $\mathscr{S}$ has a natural numbers object.

Proof. By hypothesis, we have a (generic) $T$-model $\mathscr{G}=(G, 0,1)$ in $\mathscr{E}$ and a geometric morphism $p: \mathscr{E} \rightarrow \mathscr{I}$ such that, whenever $f: \mathscr{F} \rightarrow \mathscr{F}$ is a geometric morphism and $\mathscr{A}$ is a $T$-model in $\mathscr{F}$, then there is a geometric morphism $g: \mathscr{F} \rightarrow \mathscr{E}$ such that $g^{*}(\mathscr{G})$ is isomorphic to $\mathscr{A}$ and $p g$ is naturally isomorphic to $f$. (The hypothesis actually contains more information than this, but this is all that we shall need.)

Since both the direct and inverse image functors of a geometric morphism preserve 1 , they send $T$-models to $T$-models. In particular, we have $T$-models 
$p_{*}(\mathscr{G})$ in $\mathscr{S}$ and $p^{*} p_{*}(\mathscr{G})$ in $\mathscr{E}$. The counit $\epsilon$ of the adjunction between $p^{*}$ and $p_{*}$ provides a morphism $\epsilon_{G}: p^{*} p_{*}(G) \rightarrow G$, and the naturality of $\epsilon$ implies that $\epsilon_{G}$ is a homomorphism of $T$-models $p^{*} p_{*}(\mathscr{G}) \rightarrow \mathscr{G}$.

Now let $\mathscr{A}$ be any $T$-model in $\mathscr{S}$. Applying to $\mathscr{A}$ the first paragraph of this proof, with $\mathscr{S}$ as $\mathscr{F}$ and the identity on $\mathscr{S}$ as $f$, we obtain a geometric morphism $g: \mathscr{I} \rightarrow \mathscr{E}$, an isomorphism $i: g^{*}(\mathscr{G}) \rightarrow \mathscr{A}$, and a natural isomorphism $v: \operatorname{Id} \rightarrow$ $(p g)^{*}=g^{*} p^{*}$. Applying $g^{*}$ to the homomorphism $\epsilon_{G}: p^{*} p_{*}(\mathscr{G}) \rightarrow \mathscr{G}$ and composing with these isomorphisms, we obtain the following homomorphism of $T$-models in $\mathscr{S}$ :

$$
p_{*}(\mathscr{G}) \underset{v_{p_{*}(G)}}{\longrightarrow} g^{*} p^{*} p_{*}(\mathscr{G}) \underset{g^{*}\left(\epsilon_{G}\right)}{\longrightarrow} g^{*}(\mathscr{G}) \underset{i}{\longrightarrow} \mathscr{A}
$$

(That $v_{p_{*}(G)}$ preserves the $T$-model structure follows immediately from the naturality of $v$ and the fact that $(p g)^{*}$ preserves 1 .) Since $\mathscr{A}$ was an arbitrary $T$-model in $\mathscr{S}$, we have shown that $p_{*}(\mathscr{G})$ is a weak natural numbers object in $\mathscr{P}$. By the lemma, $\mathscr{S}$ has a natural numbers object.

To complete the proof of the main theorem, we must construct a classifying topos for $T$ over $\mathscr{S}$, given an object classifier $\mathscr{S}[U]$ over $\mathscr{S}$. Although the construction is well-known, we include a description for the sake of completeness. It proceeds in three steps.

First, let $P$ in $\mathscr{S}[U]$ be the object of $K$-finite subsets of $U \times U$, where $U$ is the generic object in $\mathscr{S}[U]$. Partially order $P=K(U \times U)$ by the inclusion relation inherited from the power set of $U \times U$.

Consider an arbitrary topos $\mathscr{F}$ defined over $\mathscr{f}[U]$ by a geometric morphism $h: \mathscr{F} \rightarrow \mathscr{S}[U]$, and let $A=h^{*}(U)$ be the object of $\mathscr{F}$ classified by $h$. Invoking the left-exactness of $h^{*}$ and Lemma 6.44 and Theorem 9.16 of [4], we find that $h^{*}(P)$ is canonically isomorphic to $K(A \times A)$, partially ordered by inclusion. By Diaconescu's Theorem [2] (or see [4], Theorem 4.34), the category of geometric morphisms from $\mathscr{F}$ to $\mathscr{S}[U]^{P}$ over $\mathscr{S}[U]$ is equivalent to the category of flat internal presheaves on $h^{*}(P)=K(A \times A)$ in $\mathscr{F}$. But flat presheaves on a poset (and their morphisms) are simply the ideals in that poset, i.e., the inhabited, downward-closed, upward directed subsets of that poset (and their inclusions). When the poset has the form $K(X)$ ordered by inclusion, then the ideals correspond bijectively to the subsets of $X$, the correspondence being that an ideal $I \subseteq K(X) \subseteq \mathscr{P}(X)$ corresponds to its union $\bigcup_{p \in I} p$ and that a subset $Y$ of $X$ corresponds to the ideal $K(Y) \subseteq K(X)$. Thus, morphisms from $\mathscr{F}$ to $\mathscr{S}[U]^{P}$ over $\mathscr{S}[U]$ are equivalent to subobjects of $A \times A$, i.e., binary relations on $A$.

Now consider a topos $\mathscr{F}$ defined over $\mathscr{S}$ (not $\mathscr{F}[U]$ ) by a geometric morphism $f: \mathscr{F} \rightarrow \mathscr{S}$. A geometric morphism $g$ over $\mathscr{S}$ from $\mathscr{F}$ to $\mathscr{S}[U]^{P}$ (where the latter is an $\mathscr{S}$-topos by virtue of the composite $\mathscr{S}[U]^{P} \rightarrow \mathscr{S}[U] \rightarrow \mathscr{S}$ ) is also a morphism 
over $\mathscr{S}[U]$ if we regard $\mathscr{F}$ as defined over $\mathscr{S}[U]$ by the composite morphism $\mathscr{F} \underset{g}{\rightarrow} \mathscr{S}[U]^{P} \rightarrow \mathscr{S}[U]$. To match the notation of the previous paragraph, we call this morphism $h$ and we call the object $h^{*}(U)$ of $\mathscr{F}$ that it classifies $A$. Thus, geometric morphisms $\mathscr{F} \rightarrow \mathscr{S}[U]^{P}$ over $\mathscr{S}$ are equivalent to pairs consisting of a morphism $h: \mathscr{F} \rightarrow \mathscr{S}[U]$ over $\mathscr{S}$ and a morphism $\mathscr{F} \rightarrow \mathscr{S}[U]^{P}$ over $\mathscr{S}[U]$; these, in turn, are equivalent to pairs consisting of an object $A$ of $\mathscr{F}$ (since $\mathscr{S}[U]$ is an object classifier) and a binary relation $R \subseteq A \times A$ (by the preceding paragraph). This completes the first step of the construction: we have obtained a classifying topos for objects with a binary relation.

For the second step, we apply Example 3.59(iv) of [4] to obtain the smallest topology $j$ in $\mathscr{S}[U]^{P}$ for which the first projection of the generic binary relation, $R \subseteq U \times U \rightarrow U$, becomes an isomorphism in the sheaf subtopos. Aplying [4] Corollary 4.19 (just as in one of the usual constructions of classifying topoi, [4], 6.56), we find that a geometric morphism $g: \mathscr{F} \rightarrow \mathscr{S}[U]^{P}$, classifying an object $A$ and relation $R \subseteq A \times A$, factors through the sheaf subtopos $\mathscr{S}[U]_{j}^{P}$ if and only if $R$ is the graph of a morphism $A \rightarrow A$. Thus, we have a classifying topos for objects with an endomorphism.

Finally, passing from the topos $\mathscr{S}[U]_{j}^{P}$ to its slice topos over the generic object (the $j$-sheaf associated to the constant diagram on $P$ with value $U$ ), we obtain, as in [4] Example 4.37(i), a classifying topos for objects with an endomorphism and a global point, i.e., for $T$-models. This completes the proof of the main theorem.

Remarks. 1. In deducing the theorem from the proposition, we showed somewhat more than was strictly needed. The proposition did not really use that $\mathscr{G}$ is generic, only that it is weakly generic in the sense that every $T$-model in any $\mathscr{S}$-topos is obtainable as the inverse image of $\mathscr{G}$ under some geometric morphism over $\mathscr{S}$. Thus, to obtain the theorem, it would have sufficed to produce an $\mathscr{S}$-topos containing such a weakly generic $T$-model, given an object classifier.

2. The $T$-model $p(\mathscr{G})$, shown to be a weak natural numbers object in the proof of the proposition, is in fact a natural numbers object. Indeed, once we know, by the proposition, that $\mathscr{S}$ has a natural numbers object, we know, by [5], that the classifying topos for $T$-models is the topos of internal diagrams in $\mathscr{S}$ on the internal category of finitely presented $T$-models and that the generic $T$-model is the "inclusion" functor. This makes it easy to compute that $p_{*}(\mathscr{G})$ is the initial $T$-model.

\section{REFERENCES}

[1] J. BÉNABOU, Théories relatives à un corpus, C.R. Acad. Sci. Paris 281 (1985) pp. A831-834.

[2] R. Diaconescu, Change of base for toposes with generators, J. Pure Appl. Alg. 6 (1975) pp. 191-218. 
[3] P. FreYd, Aspects of topoi, Bull. Austral. Math. Soc. 7 (1972) pp. 1-76 and 467-480.

[4] P. Johnstone, Topos Theory, Academic Press, 1977

[5]. P. Johnstone and G. Wratth, Algebraic theories in toposes, in Indexed Categories and Their Applications, eds. P. Johnstone and R. Paré, Springer Lecture Notes in Math. 661 (1978) pp. 141-242.

[6] M. TIERNEY, Forcing topologies and classifying topoi, in Algebra, Topology and Category Theory: a collection of papers in honor of Samuel Eilenberg, eds. A. Heller and M. Tierney, Academic Press, 1976, pp. 189-210.

Mathematics Department The University of Michigan Ann Arbor, Michigan 